Occupational Optometry Clinic, and if required is referred to an ophthalmologist.

Results Based on the screening criteria, 20000 employees are eligible for the program. Approximately 3000 employees are scheduled for screening annually. Nearly $60 \%$ of enrolled employees are assessed per department. Many of those screened require health awareness related to ocular dryness and prescription-related changes. In addition early vision changes were found to be due to diabetes, cataracts, and glaucoma. About 50 annually require specialist referral.

Discussion Using a visual task analysis, the program has improved visual deficiency at Saudi Aramco. Prior to the program, many participants had not been aware of a problem. In the poster, three cases (early onset glaucoma, diabetic retinopathy, and retinitis pigmentosa) where early diagnosis and intervention prevented permanent visual impairment will be discussed.

\section{RISK OF PROSTATE CANCER AMONG FIREFIGHTERS: A REVIEW AND META-ANALYSIS OF STUDIES PUBLISHED AFTER 2007}

${ }^{1}$ IS Mehlum*, ${ }^{1} \mathrm{HA}$ Johannessen, ${ }^{2} \mathrm{~K}$ Kjærheim, ${ }^{2} \mathrm{TK}$ Grimsrud, ${ }^{1} \mathrm{KC}$ Nordby. ${ }^{1}$ National Institute of Occupational Health (STAMI), Oslo, Norway; ${ }^{2}$ Cancer Registry of Norway, Oslo, Norway

\subsection{6/oemed-2018-ICOHabstracts.1109}

Introduction Prostate cancer is the most common cancer among men; however, little is known about its aetiology. Several studies have indicated that firefighters have an increased risk, but the results have not been consistent. Firefighters are exposed to many harmful substance in their work, some of which are carcinogenic. Two reviews with meta-analyses, including studies through 2003 and 2007, respectively, found that firefighters had approximately $30 \%$ elevated risk of prostate cancer. Our objective was to perform an updated review and meta-analyses of studies published after 2007.

Methods We performed a literature review of original articles of prostate cancer in firefighters, published between January 2008 and May 2017. For the meta-analysis, we selected relevant longitudinal studies and calculated summary risk ratios (sumRRs).

Result Six studies, published 2008-2016, with a total of 5097 prostate cancer cases, were selected for the analysis; four were cohort studies and two case-control. Eight risk estimates were included in the meta-analysis, seven incidence based, one mortality based. All showed weak to moderate positive associations between firefighting and prostate cancer, although some not statistically significant. Meta-analysis of five incidence based risk estimates from cohort studies, gave sumRR 1.20 (95\% CI: 1.05 to 1.36 ). Results based on the two case-control incidence studies gave sumRR 1.24 (0.90-1.70). When all eight risk estimates were included in the meta-analysis, sumRR was 1.19 (1.08-1.32). Further analyses, however, showed significant heterogeneity between the studies.

Discussion The results showed that firefighters had approximately 20\% increased risk of prostate cancer, somewhat lower than the previous two meta-analyses found. However, occupational title may be an inaccurate measure of carcinogenic exposures and thus lead to underestimation of the cancer risk. On the other hand, reasons other than a causal relationship, particularly surveillance bias, caused by regular health examinations among firefighters, cannot be excluded. 59 POLYCHLORINATED BIPHENYLS AND DEPRESSION -
FIRST HINTS FOR A PATHOMECHANISM VIA THE THYROID AND DOPAMINE SYSTEM IN HUMANS

${ }^{1} \mathrm{PM}$ Gaum*, 1,2M Gube, 'A Esser, ${ }^{1} \mathrm{~T}$ Schettgen, ${ }^{1,3} \mathrm{~N}$ Soares Quinete, ${ }^{1,4} \mathrm{FM}$ Putschoegl, ${ }^{1} \mathrm{~T}$ Kraus, ${ }^{1 \mathrm{~J}}$ Lang. 'Institute of Occupational Medicine, University Hospital RWTH Aachen, Germany; ${ }^{2}$ Health Office of the City and Area of Aachen, Germany; ${ }^{3}$ Florida International University (FIU), Florida, Miami, USA; ${ }^{4}$ Department of Psychiatry and Psychotherapy, Central Institute of Mental Health, Mannheim, Germany

\subsection{6/oemed-2018-ICOHabstracts.1110}

Introduction After PCB (polychlorinated biphenyls) exposure the development of depression has been described (Fitzgerald, et al. 2008). In general, depressive symptoms are associated with lower dopamine concentration and disturbed thyroid function. Thyroxin (T4) is necessary for dopamine synthesis in the brain (Hassan, et al. 2013). Bound to transthyretin (TTR); T4 is transported into the brain. Since PCB can displace T4 by binding to TTR itself (Hamers, et al. 2011); the concentration of free T4 (fT4) increases under PCB exposure. This study investigates the interaction of $\mathrm{PCBs}$ and $\mathrm{fT} 4$ related to the dopamine metabolite homovanillic acid (HVA) as well as to depression in humans.

Methods This study is part of the HELPcB (Health Effects in high Level exposure to $\mathrm{PcB}$ ) surveillance program. Altogether, 109 occupationally exposed individuals $[\mathrm{m}=101$ (92.7\%); age: mean $=44, \mathrm{SD}=12.8$ ] participated at three yearly assessments. Individuals with thyroid- or dopamine-relevant medication were excluded. PCBs were measured in plasma $(\mu \mathrm{g} / \mathrm{L})$, fT4 in serum, HVA in urine and depression was assessed with the PHQ-9 (Löwe, et al. 2002). PCB-congeners were summed up to LPCB (lower-chlorinated), HPCB (higher-chlorinated) and dIPCB (dioxin-like). A sum variable was generated for the PHQ-9. The interactions of PCBs and fT4 related to HVA and depression were tested with mixed models.

Result Significant interactions related to HVA were found for all PCB-subtypes (e.g. LPCB: $B=-0.5, p<0.01$ ). Under high PCB-exposure high fT4 levels are associated with a lower HVA concentration and vice versa. The interaction related to depression was only significant for LPCB $(B=0.1, p<0.01)$. More depressive symptoms were found for high PCB-exposure with increasing fT4 level and vice versa.

Discussion The interactions related to HVA support the postulated pathomechanism via TTR. Individual and environmental factors may be a reason that the interactions for the behavioural outcome of depressive symptoms were only partially confirmed.

\section{ANNUAL CHECK-UP: A RELEVANT TOOL FOR WORKER'S WELLBEING AND WORKPLACE HEALTH PROMOTION}

Cheikh AKA Cisse. Private part time Occupational Physician, Dakar, Senegal

\subsection{6/oemed-2018-ICOHabstracts.1111}

Introduction Annual check-up is one important component of periodical medical examination legally instituted by labour code in workers' health surveillance. It allows the assessment of the health status of the worker in order to renew his/her medical fitness to his/her job and to detect any illness related or not to work. Thus the outcomes obtained help us to design appropriate action plan for both prevention of occupational hazards and health promotion. 